\title{
Gerd Rudel \\ Marxistische Staatstheorie und sozialistische Strategie in der Bundesrepublik
}

\section{Vorbemerkung}

Der Zusammenhang zwischen der theoretischen Einschätzung des bürgerlichen Staates in seinen verschiedenen Erscheinungsformen und der Formulierung einer adäquaten sozialistischen Strategie ist offenkundig. Der entscheidende strategische Stellenwert materialistischer Staatstheorie ist deshalb unter Marxisten unstrittig. Nicht von ungefähr war das Staatsproblem $\mathrm{ja}$ in all jenen Phasen aktuell, in denen es sich für gesellschaftliche Oppositionsbewegungen als Existenzfrage erwies, ihre konkrete Politik in bzw. gegenüber dem bürgerlichen Staat theoretisch zu fundieren.

Wenn in diesem Aufsatz die materialistische Binsenweisheit des Zusammenhangs von Staatstheorie und sozialistischer Politik als Problematik erscheint, die es systematisch aufzurollen gilt, so hat dies seine Ursache in einem paradoxen Sachverhalt. Auf der einen Seite gab es in den siebziger Jahren eine äußerst ausführliche Debatte um eine »Ableitung« des bürgerlichen Staates. Ein wesentliches Kennzeichen dieser Diskussion war - soviel schon vorab - ein erstaunliches Maß an Abstinenz in Fragen sozialistischer Strategie. Heute - zu Beginn der achtziger Jahre - stellt sich die Situation gewissermaßen umgekehrt dar: Dem Anwachsen von diversen Oppositionsansätzen, denen es um die konkrete und aktuelle Veränderung ihrer Lebensumstände geht, korrespondiert keineswegs eine staatstheoretisch fundierte Strategiedebatte. Im Gegenteil, theoretische Auseinandersetzungen - soweit sie überhaupt noch geführt werden - um eine adäquate sozialistische Strategie drehen sich im Kern um die alte Streitfrage »neue sozialistische Partei versus außerparlamentarischer Kampf«, als ob die bundesdeutsche Linke ihre Erfahrungen und Debatten im Hinblick auf dieses allerdings entscheidende Problem sozialistischer Politik vergessen oder verdrängt hätte.

Wie nun, soll hier für die Wiederaufnahme der $1977 / 78$ sanft entschlummerten und allzu häufig als »toter Hund« gebrandtmarkten »Staatsableiterei« plädiert werden? Keineswegs, aber in einer gesellschaftlich-politischen Situation, in der die erwähnten Oppositionsansätze vor der Alternative Integration oder Marginalisierung/Kriminalisierung stehen, erscheint mir notwendig zu sein, darauf hinzuweisen, daß das verbreitete spontaneistische Gefühl »Null Bock auf den Staat « eine theoretisch fundierte Analyse des bürgerlichen Staates und eine - daraus abgeleitete - sozialistische Strategie nicht ersetzen kann. Der um sich greifende anti-theoretische Affekt, die Weigerung, »überhaupt noch die 'Mühe des Begriffs' auf sich zu nehmen« (Kraushaar 1978, 30), die stillschweigende Ablehnung »altlinker Hirnwichser « (vgl. dazu Spengler 1981, 180) ist ein Indiz dafür, daß Ende der siebziger Jahre eine Entwicklung eingesetzt hat, die als geradezu typisch für die Geschichte der bundesdeutschen Linken angesehen werden muß: die umstandslose Distanzierung von der eigenen theoretischen, organisatorischen und strategischen Vergangenheit (in unserem Falle

von Seminarmarxismus, Staatsableiterei, Theoriearbeit, K-Gruppen-Dogmatismus etc.), ohne daß diese Vergangenheit und ihre Defizite wenigstens produktiv aufgearbeitet worden wären. Wieder einmal scheint sich die Linke in der BRD als unfähig zu erweisen, aus ihrer Geschichte zu lernen. 
Das Ziel dieses Aufsatzes ist es deshalb, die mehr oder minder eingeschlafene staatstheoretische Diskussion voranzutreiben, indem deren oft genug vernachlässigter strategischer Aspekt akzentuiert wird. Dies soll auf zweierlei Weise geschehen: Einmal soll in einem Überblick über wichtige staatstheoretische Ansätze ${ }^{1}$ die Frage gestellt werden, inwieweit sich diese Ansätze den politischen und strategischen Herausforderungen in bestimmten gesellschaftlichen Problemkonstellationen gestellt haben. Zum anderen erhoffe ich mir von den Ergebnissen einer derartigen Analyse zumindest einige Hinweise auf die Aufgaben künftiger staatstheoretischer Bemühungen, denen die Beteuerung der strategischen Relevanz marxistischer Staatstheorie nicht nur ein Lippenbekenntnis ist.

\section{Wolfgang Abendroth: Gesellschaftskritik und Verfassungstheorie im politischen Kampf der Ära Adenauer}

Mit seinem »einzelkämpferischen« Festhalten an Grundpositionen des Marxismus war Wolfgang Abendroth im militant antikommunistischen Klima der Adenauer-Ära von vornherein ein Außenseiter. Angesichts der Rekonstruktion des Kapitalismus in der BRD und der sukzessiven Beschneidung grundgesetzlich verbriefter Grundrechte in der autoritären Kanzlerdemokratie Adenauers ging es Abendroth nicht in erster Linie um die Grundlegung einer materialistischen Staats- und Verfassungstheorie. Er betrachtete es vielmehr als seine vordringliche wissenschaftliche Aufgabe, die Möglichkeit einer sozialistischen Transformation der kapitalistischen Gesellschaft auf dem Wege über die in der bundesrepublik nach dem Kriege installierte parlamentarische Demokratie theoretisch zu untermauern.

Abendroths Argumentation war aufgrund dieser Konstellation im wesentlichen defensiv und hatte zwei Aspekte: Zum einen wandte sich Abendroth in seiner Verfassungsinterpretation gegen jene Deutungen des Grundgesetzes, die die bestehende politisch-gesellschaftliche Realität insbesondere des kapitalistische Wirtschaftssystem als einzig verfassungsmäßig hinzustellen versuchten (vgl. A bendroth 1975, S.64-69). Gegenüber diesen Apologeten der herrschenden Verhältnisse verwies Abendroth auf den Kompromißcharakter des Grundgesetzes (vgl. Abendroth 1966, S.37-41). Er interpretierte die bundesrepublikanische Verfassung als das Produkt eines Kompromisses zwischen den Kräften im Parlamentarischen Rat, die nach 1945 eine Restauration der kapitalischen Gesellschaft anstrebten, und jenen, deren Ziel die demokratisch-sozialistische Umgestaltung von Wirtschaft und Gesellschaft war. Durch die Festschreibung dieser politisch-sozialen Kräftekonstellation unmittelbar nach dem Kriege im Grundgesetz sah Abendroth die konkrete Ausgestaltung der gesamtgesellschaftlichen Ordnung prinzipiell offengehalten. Theoretische Grundlage dieser Verfassungsinterpretation war die Unterscheidung zwischen demokratischer Verfassungsordnung und bürgerlichem Staatsapparat, die Abendroth nicht unmittelbar gleichgesetzt wissen wollte (vgl. Abendroth 1975, S.165). Auf der Basis dieser Unterscheidung nämlich konnte er gegenüber der restaurierten kapitalistischen Gesellschaft die im Grundgesetz angelegte Verfassungsmäßigkeit einer sozialistischen Transformation der BRD geltend machen.

Zum anderen griff Abendroth mit seiner wissenschaftlichen Arbeit direkt in den politischen Kampf der Adenauer-Ära ein. Ziel seiner gesellschaftskritischen Analysen, die er ganz offensichtlich als Moment sozialistischer Strategie begriff (vgl. Perels 1974, S. 147), war es, die politische Demokratie als mögliches Transformationsfeld zum Sozialismus ge- 
gen die Bedrohung durch ein neues faschistisches oder autoritäres System zu verteidigen. In diesem Sinne ging es ihm vermittels des Kampfes um Rechtspositionen vor allem um die Erhaltung formaldemokratischer Verfassungsnormen.

Politisch-strategischer Adressat seiner verfassungstheoretischen und gesellschaftskritischen Analysen war die Arbeiterbewegung, insbesondere die SPD, die er trotz aller kritischen Distanz zu ihrer aktuellen Politik als einzig mögliche Operationsbasis sozialistischer Zielvorstellungen betrachtete (vgl. A bendroth 1976, S. 253). Bis zu seinem Ausschluß aus der Partei im Jahre 1961 versuchte er deshalb in stetigem Kampf gegen die Anpassung der SPD an bürgerliche Ideologien, den sozialdemokratischen Flügel der Arbeiterbewegung an sein sozialistisches Erbe zu erinnern und die marxistische Tradition der SPD wachzuhalten.

Abendroths wissenschaftliche und politische Bedeutung liegt einerseits in der politologisch und verfassungsrechtlich untermauerten Verteidigung bürgerlich-demokratischer Freiheitsrechte und der rechtsstaatlich verfaßten politischen Demokratie im antikommunistischen Klima des Kalten Krieges. Zum anderen zeichnet ihm sein Gespür für die Relevanz politischer Kräftekonstellationen und die Orientierung seiner Theoriebildung an den strategischen Problemen gesellschaftsverändernder Praxis aus. Ungeachtet der verschiedentlich geäußerten Kritik, Abendroths Position sei legalistisch und letztlich idealistisch, weil Abendroths immanent juristische und politisch defensive Argumentation zu einer Verrechtlichung und damit einer Entpolitisierung sozialistischer Strategie führe ${ }^{2}$, ist Abendroths theoretische Grundidee politisch-strategisch unverzichtbar. Angesichts der gegenwärtigen ökonomischen Krise mit stagnierenden Reallöhnen, Arbeitsintensivierung und Massenarbeitslosigkeit, angesichts des massiven Abbaus demokratischer Grundrechte und der Zunahme staatlicher Repression ist die von Abendroth formulierte Strategie der Verteidigung rechtsstaatlich-demokratischer Verfassungspositionen und damit ja auch des Spielraums der Linken in der BRD von aktueller Relevanz.

\section{Die "Frankfurter Schule«: Kritische Theorie und Praxisabstinenz}

Außer Wolfgang Abendroth gab es nach der Wiedereröffnung des »Instituts für Sozialforschung in Frankfurt im Jahre 1951 einen weiteren Traditionsstrang kritischer Theoriebildung in der BRD der Nachkriegszeit. Doch während - wie wir gesehen haben - Abendroth den Typus des in der Arbeiterbewegung verwurzelten und an den Erfordernissen sozialistischer Politik orientierten Wissenschaftlers repräsentierte, entsprachen die Theoretiker der „Frankfureter Schule« in hohem Maße der landläufigen Vorstellung von »freischwebenden Intellektuellen«. Die selbstgewählte Distanz von Adorno und Horkheimer gegenüber aktuellen und potentiellen Oppositionsansätzen in der Adenauer- ̈̈ra blieb selbstverständlich nicht ohne Auswirkungen auf die Theoriebildung der Frankfurter Schule.

Die Abwendung des Kreises um Horkheimer und Adorno von den Ursprüngen des Institus für Sozialforschung kam schon in der Tatsache zum Ausdruck, daß sie dessen Tradition der Erforschung der Arbeiterbewegung und der Weiterentwicklung des Marxismus nach dem Krieg kaum fortsetzten. Im Gegenteil: Die im wesentlichen schon während des amerikanischen Exils vollzogene Abkebr von der marxistischen Theorietradition und der Übergang zur geschichtsphilosophischen Kritik der gesamten abendländischen Aufklärungstradition, in die implizit auch das Marxsche Werk gestellt wurde (vgl. Jay 1976, S. 303), wurde nach der Rückkehr nach Frankfurt bekräftigt. 
Zwar waren vor allem Adornos subtile Analysen der spätkapitalistischen "Kulturindustrie", die von der Frankfurter Schule als Ursache der effektiven Stabilisierung bürgerlicher Herrschaft mittels direkter Bewußtseinsformierung angesehen wurde (vgl. Mirbach, 1979, S. 152 - 170), immer noch von einer immensen Schärfe und Radikalität des theoretischen Zugriffs. Doch gegenüber dieser Kritik jener systemstabilisierenden Mechanismen, die in der Perspektive der »Kritischen Theorie« eine emanzipatorische Revolution verhinderten, kam die Untersuchung der konkreten politischen und ökonomischen Strukturen der BRD entschieden zu kurz. Verhängnisvoll wirkte sich vor allem das Defizit der Frankfurter Schule auf der Ebene der politischen Theorie aus. Im Gegensatz zu Abendroth befaßten sich Adorno und Horkheimer - wenn überhaupt - nur höchst unzureichend mit den Strukturen der bürgerlichen Demokratie und den in den bürgerlichen Freiheitsrechten implizierten Ansatzpunkten einer emanzipatorischen Strategie.

Die Frage nach den Möglichkeiten und Schwierigkeiten einer sozialistischen Politik in der Zeit des Kalten Krieges stand jedenfalls für die kritischen Theoretiker der Frankfurter Schule niemals zur Debatte. Ihrer radikalen Kulturkritik entsprach keine radikale Praxis. Dies um so weniger, als Adorno und Horkheimer den Träger einer derartigen Praxis nicht zu erkennen vermochten. Die »Kritische Theorie« bezweifelte nicht nur die Rolle der angeblich in den bürgerlichen Herrschaftszusammenhang integrierten Arbeiterklasse als politisch handlungsfähiges Subjekt, sondern sie negierte vielmehr überhaupt die mögliche Existenz eines revolutionären Potentials, das in der Lage sei, eine emanzipatorische Veränderung der kapitalistischen Gesellschaft herbeizuführen (vgl. Jay 1976, S. 325, 339, 343).

Offensichtlich wirkte sich die die Adenauer-Ära kennzeichnende politische Stagnation und gesellschaftliche Immobilität derart lähmend auf die analytische Sensibilität der Frankfurter Schule aus, daß sie vorhandene Widerspruchspotentiale übersah und deshalb die relative Stabilität hochentwickelter kapitalistischer Gesellschaften wie der BRD und der USA zu einem raum-zeitlich übergreifenden Phänomen hypostasierte. In freiwilliger Isolation von allen gesellschaftlichen und politischen Oppositionsansätzen standen Horkheimer und Adorno der Realität des restaurierten kapitalistischen Systems in der BRD zwar kritisch, jedoch politisch-strategisch bilflos und deshalb letztlich resignativ und passiv gegenüber. Ihre emphatische Beschwörung des Ziels einer emanzipierten Gesellschaft stand somit in einem krassen Gegensatz zu ihrer eklatanten Unfähigkeit, handlungsorientierendes Wissen zu vermitteln und damit einen strategisch-praktischen Weg zu eben dieser Gesellschaft aufzuzeigen.

Trotz dieses Widerspruchs von radikaler kritischer Theoric und völliger Praxisabstinenz in der Frankfurter Schule war das Institut für Sozialforschung eines der wenigen Institute, das überhaupt zu kritischem Denken anleitete, wenn nicht sogar der wichtigste »akademische Kristallisationspunkt philosophischer und sozialwissenschaftlicher Gesellschaftskritik (Wolf 1973, S. 38) in der BRD. Die "Kritische Theorie« hatte deshalb erheblichen Einluß auf die konzeptionelle Neuorientierung gesellschaftskritischer Analysen in der antiautoritären Studentenbewegung, ja sie wurde zum »Sozialisationsmedium《 einer ganzen Generation kritischer Sozialwissenschaftler (vgl. Dubiel 1978, S. 212). 


\section{Johannes Agnoli: Plädoyer für eine außerparlamentarische Fundamentalopposition}

Waren Abendroths gesellschaftskritische Konfrontation von Verfassungsnorm und Verfassungswirklichkeit sowie die Kulturkritik der Frankfurter Schule während der Ära Adenauer in einem Umfeld sozialwissenschaftlicher Disziplinen, denen es wesentlich um die ideologische Legitimation und Stabilisierung der bestehenden Machtverhältnisse ging, im Prinzip ${ }^{3}$ die einzigen kritischen Theorieansätze, so kam es im Laufe der sechziger Jahre zu einer quantitativen und qualitativen Entfaltung materialistischer Theoriebildungsversuche in einem bislang nicht gekannten Ausmaß. Den unmittelbaren politischen Anlaß für eine derartige Intensivierung und Radikalisierung systemkritischen Denkens stellte die 1966 von CDU/CSU und SPD gebildete "Große Koalition « dar. Nachdem die beginnende Auflockerung der Fronten im Ost-West-Konflikt die Wirksamkeit des aggressiven Antikommunismus der Adenauer-Ära beeinträchtigt hatte und Spiegel-Affäre und Rezession das bislang so stabile ideologische Gebäude des christdemokratischen Nachkriegsdeutschland nachhaltig erschüttert hatten, symbolisierte die Große Koalition ganz offenkundig die Diskrepanz zwischen dem demokratischen Anspruch der BRD und der undemokratischen und gesellschaftlichen Realität des Landes. Die Große Koalition verkörperte den Versuch der Institutionalisierung jener autoritären und etatistischen Tendenzen zu einer "formierten Gesellschaft ", mit der die Unionsparteien die ökonomische, politische und ideologische Krise in den sechziger Jahren in den Griff zu bekommen hoffte. Verstärkt durch die Auseinandersetzungen um die Verabschiedung der Notstandsgesetze geriet immer stärker das gesamte innenpolitische Macht- und Herrschaftsgefüge in der BRD und sein Zusammenhang mit der kapitalistischen Wirtschaftsstruktur in den Blickpunkt des analytischen Interesses systemkritischer Intellektueller (vgl. Krippendorf 1971, S. 113).

Johannes Agnolis Versuch, Parlamentarismusund Kapitalismuskritik miteinander zu verbinden, und seine Bemühungen, staatstheoretische Überlegungen materialistisch zu fundieren, sind ein Beispiel für diese Entwicklungsphase marxistisch orientierter Theoriebildung in der BRD. Agnoli unternahm es nämlich, den spätestens mit der Bildung der Großen Koalition als Fehlentwicklung der parlamentarischen Demokratie allseits beklagten Funktionsverlust, das heißt den Verlust an faktischer politischer und legislativer Entscheidungskompetenz des Parlaments, als funktional notwendig für den Bestand hochentwickelter kapitalistischer Gesellschaften aufzuweisen. Die grundsätzliche Funktion der parlamentarischen Demokratie sah Agnoli in der Stabilisierung des Kapitalismus durch den Versuch, den gesellschaftsstrukturell bedingten antagonistischen Klassenwiderspruch zu glätten und sozial auszugleichen (vgl. Agnoli 1968 a, S. 9f.): Mit Hilfe einer Politik des sozialen Ausgleichs werde der durch die kapitalistische Produktionsweise bedingte Klassenwiderspruch auf einen pluralistischen Interessenkonflikt, auf einen Konflikt um die Verteilung des Sozialprodukts reduziert. In diesem manipulativen Prozeß der Verschleierung der realen Macht- und Abhängigkeitsverhältnisse spielte für Agnoli das Parlament eine entscheidende Rolle. Zwar sei es als eine die Souveränität des Volkes verwirklichende Körperschaft faktisch bedeutungslos geworden, als Konstitutionalisierungsorgan der Beschlüsse der herrschenden Oligarchien aber trage es wesentlich zur Stabilisierung der kapitalistischen Gesellschaft bei (vgl. ebd., S. 63).

Die strategischen Konsequenzen Agnolis aus dieser Analyse des bürgerlichen Parlamentarismus waren eindeutig: Sollte der durch das Parlament und die »Volksparteien« verschleierte gesellschaftliche Antagonismus wieder sichtbar gemacht, der Klassenkampf aktuali- 
siert werden, dann bedurfte es einer Fundamentalopposition, die sich bewußt auf außerparlamentarische Aktionsformen konzentrieren sollte (vgl. Agnoli 1968 b, S. 47). Nur so könnten die Massen politisiert, das heißt über die Unvernünftigkeit von Herrschaft und Ausbeutung aufgeklärt werden (vgl. Agnoli 1968 a, S. 75). Nachdrücklich warnte Agnoli vor der Domestizierung einer derartigen Fundamentalopposition durch deren mögliche Parlamentarisierung:

"Die fundamentaloppositionellen Parteien, die sich auf das parlamentarische Spiel einlassen und den außerparlamentarischen Kampf nicht mehr als das wesentliche Mittel des Herrschaftskonflikts praktizieren, drohen ihre emanzipatorische Qualität zu verlieren und sich in bürokratische Integrationsapparate zu verwandeln.« (Agnoli 1968 b, S. 29)

Agnolis Studie über die "Transformation der Demokratie« war als ein in der Form einer Verknüpfung von Parlamentarismus- und Kapitalismuskritik konzeptualisierter Versuch einer materialistischen Fundierung systemkritischer Staatstheorie gewiß ein Meilenstein in der Entwicklung des Marxismus in der BRD. Wegen ihrer politisch-strategischen Implikationen war sie zudem eine Standardquelle der Neuen Linken und eines der einflußreichsten Bücher der studentischen Protestbewegung. Doch gerade in politisch-strategischer Hinsicht waren Agnolis parlamentarismuskritischen Untersuchungen nicht unproblematisch. Da er sich bei seiner Analyse auf die BRD konzentrierte, lief er Gefahr, die politischen Zustände zur Zeit der Großen Koalition zu Gesetzmäßigkeiten der bürgerlichen Gesellschaft überhaupt zu hypostasieren. So geriet die Parlamentarismuskritik Agnolis - vor allem in der Rezeption bei weiten Teilen der studentischen Protestbewegung - unter der Hand zu einer unreflektierten Ablebnung des parlamentarischen Systems, der eine realistische Einschätzung der Möglichkeiten und Grenzen bürgerlich-liberaler Freiheitsrechte nicht gelingen konnte.

\section{Die antiautoritäre Studentenbewegung und der bürgerliche Staat}

Agnolis vehementes Plädoyer für die Konstituierung einer außßerparlamentarischen Fundamentalopposition fand ab 1965 - von Westberlin ausgehend und nach der Erschießung Benno Ohnesorgs am 2. Juni 1967 auf die gesamte BRD übergreifend - in der antiautoritären Studentenbewegung seinen realgesellschaftlichen Ausdruck. Die Verschlechterung der Studienbedingungen und die daraus resultierenden Auseinandersetzungen mit den Universitätsbürokratien, die von der "Großen Koalition« symbolisierte Krise des Parlamentarismus in der BRD, der im Springer-Konzern beispielhaft ausgedrückte Zerfall der bürgerlichen Öffentlichkeit, das brutale Eingreifen der westlichen Führungsmacht USA in Vietnam, die Wirtschaftskrise in der BRD - das Zusammentreffen all dieser gesellschaftlichen, politischen und ökonomischen Faktoren ${ }^{4}$ setzte bei Teilen der studentischen Intelligenz (vor allem in den geistes- und sozialwissenschaftlichen Fachbereichen) Lern- und Politisierungsprozesse in Gang, in deren Verlauf die Diskrepanz zwischen bürgerlicher Ideologie und der Realität dieser Gesellschaft immer deutlicher erkannt wurde.

Da sich die antiautoritäre Bewegung eigentlich von Beginn an mit der bürgerlichen Staatsgewalt konfrontiert sah und diese Konfrontation im Zuge der Radikalisierung des studentischen Protestes immer häufiger »handgreifliche« Formen annahm, standen Versuche, das Phänomen Staat theoretisch in den Griff zu bekommen, im Mittelpunkt der analytischen Bemühungen der Studentenbewegung. Zwar kann keine Rede davon sein, es hätte je eine 
einheitliche, allseits akzeptierte und theoretisch stringente Staatstheorie der Studentenbewegung gegeben. Dazu war diese Bewegung in sich zu heterogen, Versuche zu einer theoretischen Vereinheitlichung zu diskontinuierlich. Neben Agnolis Parlamentarismuskritik und diese teilweise aufnehmend - war vor allem eine Theorievariante einflußreich, die von Vertretern des konsequent antiautoritären Flügels des SDS wie Hans-Jürgen Krahl und Rudi Dutschke entwickelt wurde. In Anknüpfung an bestimmte Theoreme der Kritischen Theorie ${ }^{5}$ war das Staatsverständnis des anitautoritären Flügels von der Überzeugung bestimmt, die BRD verkörpere einen spezifischen Typus eines »autoritären Staates«. Autoritärer Staat, das hieß in der analytischen Perspektive dieser Theoretiker: staatliche Regulierung und Lenkung der Wirtschaft im Sinne eines ökonomischen Krisenmanagements (vgl. Dutschke 1968, S. 53; Krahl 1971, S. 212); Stabilisierung des Spätkapitalismus durch ein umfassendes, schier undurchdringlich erscheinendes Netzwerk von politischer Manipulation, Indoktrination und Bedürfnissteuerung (vgl. Krahl 1971, S. 235 f.; Reiche 1968, S. 28). Krahl sprach gar von der »Allgegenwart des autoritären Staates« (Krahl 1971, S. 29), Dutschke von einem Kontinuum von Herrschaft, das alle gesellschaftlichen Gruppen in den repressiven Gesamtzusammenhang integriere (vgl. Dutschke 1968, S. 89 und 91). Vor dem Hintergrund dieses Bildes einer hermetisch abgeriegelten reaktionären Gesellschaft war es nicht weiter verwunderlich, wenn die antiautoritären Theoretiker nicht nur den bürgerlichen Staat als autoritär charakterisierten, sondern das spätkapitalistische System insgesamt als »faschistisch« bezeichneten. Eine scheinbare Bestätigung für diesen Faschismusverdacht sahen sie in der Großen Koalition, vor allem aber in den geplanten Notstandsgesetzen und in den repressiven Reaktionen des Staates und der Universitätsbürokratien auf die Protestaktionen der kritischen Studenten.

Eine derartige Qualifizierung der BRD als autoritären Staat mit faschistoiden Tendenzen konnte nicht ohne Auswirkungen auf die politische Strategie der Studentenbewegung bleiben. Wenn tatsächlich alle gesellschaftlichen Gruppen, also auch und vor allem die Arbeiterklasse in den bürgerlichen Herrschaftszusammenhang integriert waren, dann konnte sich die Hoffnung auf eine revolutionäre Veränderung der kapitalistischen Gesellschaftsformation nur noch auf Randgruppen als Initiatoren eines qualitativen gesellschaftlichen Wandels beziehen. Vor dem Hintergrund dieser Prämissen konnten die antiautoritären Theoretiker Protestbewegungen wie die studentische in der BRD als zur damaligen Zeit »einziges revolutionäres Potential der fortgeschrittenen Industriegesellschaften« (Negt 1971, S. 35 f.) interpretieren und die kritischen Studenten als eine »'Schlüsselgruppe' der antikapitalistischen Opposition« (Berndt 1968, S. 98) bezeichnen, ja ihnen sogar die Rolle einer »Avantgarde im internationalen Klassenkampf« (Nirumand 1968, S. 1-17) zuordnen. War das Staatsverständnis der antiautoritären Theoretiker also einerseits die Grundlage für eine Randgruppenstrategie, in der der studentischen Protestbewegung eine entscheidende Funktion zukam, so hatte die Einschätzung der BRD als autoritären Staat andererseits auch Auswirkungen auf die konkreten Aktionsformen der Studentenbewegung. Eine Mitarbeit in Parteien, Gewerkschaften oder sogar im Parlament schied für den antiautoritären Flügel des SDS von vornherein aus ${ }^{6}$, hätte dies doch eine Integration in den spätkapitalistischen Herrschaftszusammenhang bedeutet. Eine Aktualisierung des Klassenkampfs, die Durchbrechung der bürgerlichen Manipulationsmechanismen und die Politisierung der entmündigten Massen erwartete sich die antiautoritäre Protestbewegung vielmehr von direkten Aktionen außerparlamentarischer Aufklärung, etwa in Form provokativer Demonstrationstechniken. 
Die Problematik des Staatsverständnisses der antiautoritären Protestbewegung in theoretischer wie auch - daraus resultierend - politisch-strategischer Hinsicht ${ }^{7}$ liegen auf der Hand. Da sich die studentischen Theoretiker vornehmlich mit der Analyse der gesellschaftlichen und staatlichen Repressions- und Manipulationsmechanismen und den dadurch hervorgerufenen Entfremdungsphänomenen beschäftigten (vgl. Krüger 1973, S. 124), verzichteten sie einerseits fast völlig darauf, die ökonomischen Grundstrukturen des spätkapitalistischen Gesellschaftssystems zu untersuchen. Die der kapitalistischen Produktionsweise immanente Widersprüchlichkeit schien für die antiautoritären Theoretiker kein Thema analytischer Bemühungen mehr zu sein, klassenanalytische und ökonomiekritische Fragestellungen wurden deshalb ausgeklammert (vgl. Kukuck 1974, S. 84, Krüger 1973, S. 125). Zum anderen fehlte in den staatstheoretischen Theorieansätzen etwa von Dutschke und Krahl jeglicher Versuch, die bürgerliche Demokratie, die in ihr implizierten Freiheitsrechte und deren strukturellen Zúsammenhang mit dem kapitalistischen Gesellschaftssystem systematisch zu untersuchen. Fixiert auf die Erscheinungsformen staatlichen Handelns, insbesondere auf die gewaltsam-repressiven Reaktionen des bürgerlichen Staates gegenüber der Studentenbewegung selbst, die als Ausdruck der Verwandlung der »demokratisch legitimierten Exekutive zum faschistischen Terrorinstrument« (Lefèvre 1968, S. 12) begriffen wurden, leistete das Staatsverständnis der antiautoritären Theoretiker einer Verzerrung der analytischen Perspektive derart Vorschub, daß der angesichts der politischen Realitäten unhaltbare und zudem völlig abistorische Faschismusvorwurf eine gewissermaßen notwendige Folge war.

Vor dem Hintergrund der Qualifizierung der BRD als faschistisch konnte eine realistische Einschätzung der Möglichkeiten und Grenzen einer vornehmlich von Intellektuellen getragenen Oppositionsbewegung nicht mehr gelingen. Die antiautoritäre Studentenbewegung stand vor einer - wie sich zeigen sollte - realpolitisch perspektivlosen Alternative: entweder totale Integration in den autoritär-faschistischen Repressionszusammenhang oder radikale Revolte und absolute Verweigerung Marcusescher Prägung. Der Faschismusverdacht der antiautoritären Theoretiker muß deshalb als Ursache des Realitätsverlustes der studentischen Protestbewegung angesehen werden. Die Hypostasierung der eigenen, in ihren Auswirkungen nicht mehr selbstkritisch diskutierten Aktionen als einzig möglichen Ansatz zur Durchbrechung des repressiv-manipulativen Gesamtzusammenhangs und die daraus resultierende zunehmende Verselbständigung provokativer Demonstrationstechniken verdrängten sukzessive jene Bemühungen, durchdachte Strategiekonzepte zu erarbeiten, die auch den Stellenwert der parlamentarischen Demokratie für eine sozialistische Transformation der kapitalistischen Gesellschaft hätten einbeziehen können. Nicht von ungefähr begannen mit dem unreflektiertem Aktionismus der Studentenbewegung auch die Diskussionen über die Legitimität von Gewaltanwendung als Widerstand gegen die für faschistisch gehaltene Staatsgewalt der BRD. ${ }^{8}$

Die Radikalisierung der studentischen Protestbewegung, deren wesentliche theoretische Grundlage das Staatsverständnis der antiautoritären Theoretiker war, hatte ein eigentümlich widersprüchliches Ergebnis. Zum einen rückte Ende der sechziger Jahre zum ersten Mal in der Geschichte der BRD die revolutionäre Perspektive in den Mittelpunkt des theoretischen und strategischen Interesses der Linken, war die radikale gesellschaftsverändernde Praxis Bezugspunkt jeglicher Theoriebildung. Zum anderen trug eben diese Theorie zum zunehmenden Realitätsverlust der antiautoritären Bewegung bei, konnte es jedenfalls nicht verhindern, daß große Teile der protestierenden Intelligenz zu einer selbstkritischen 
Einschätzung der eigenen Aktionen, geschweige denn zur Entwicklung realistischer Strategiekonzepte antikapitalistischer Interessenvertretung nicht mehr fähig war.

So war es nicht verwunderlich, daß sich der gemeinsame Aktionszusammenhang, der die studentische Protestbewegung trotz aller theoretischen und organisatorischen Heterogenität zusammengehalten hatte, nach dem Scheitern zentraler Kampagnen (gegen Springer, gegen die Notstandsgesetze, gegen den Vietnam-Krieg) sukzessive auflöste. Der SDS war nicht mehr in der Lage, seine Rolle als theoretische Speerspitze und als Aktionskern der Bewegung zu spielen und die auseinanderstrebenden Strömungen auf eine gemeinsame Praxis zu verpflichten. Es kam zu einem "Entmischungsprozeß", in dessen Verlauf sich die auBerparlamentarische Opposition in verschiedene, zum Teil miteinander konkurrierende Gruppen, Organisationen und Parteien aufsplitterte. Als Resultat dieses Entmischunsprozesses ergab sich eine paradoxe Situation: Zum einen existierte als Ergebnis der von der Studentenbewegung bewirkten Politisierung ein relativ breites linkes Potential, ein Milieu, in dem eine spezifische Sensibilität für soziale Mißstände und Ungerechtigkeiten und ein Bewußtsein der Möglichkeit ihrer emanzipatorischen Veränderung zu verzeichnen war. Auf der anderen Seite konnte von einer einheitlichen linken Bewegung mit einer gemeinsamen Praxis keine Rede mehr sein. Die heillose Zersplitterung der Linken führte zum Verlust gemeinsamer Handlungsfähigkeit und zu einer Einbuße an gesellschaftlichem Einfluß.

\section{Das strategische Defizit der "Ableitungs"-Diskussion}

Nach dem Zetfall der antiautoritären Protestbewegung rückte die Staatsproblematik mit einiger Notwendigkeit in den Mittelpunkt des analytischen Interesses marxistischer Theoretiker, mußten doch die Erfahrungen der Studentenbewegung mit dem bürgerlichen Staat aufgearbeitet werden:

„Wie konnte das Gebilde Staat, das eben noch die antikapitalistisch revoltierenden Minderheiten mit Gewalt von der Straße fegte, auch weiterhin die breite Loyalität der beherrschten Gesellschaftsklassen an sich ziehen? Wie kam es, daß trotz Perfektionierung der staatlichen Unterdrückungsapparaturen, trotz einschneidenden Abbaus demokratischer Rechte, trotz offensichtlich parteiischer Intervention zugunsten der Verwertungsbedingungen westdeutscher Kapitale, wie kam es, daß trotz des mehr und mehr durchscheinenden 'klasseninstrumentellen' Charakters des Staates die breiten Arbeitermassen und proletarisierten Mittelschichten auch weiterhin loyal den Staat in seine Souveränität setzten? Wenn auch die herrschenden Ideologen sich in dieser Hinsicht alle Mühe gaben - konnte dies alles Resultat propagandistischer Lüge oder betrügerischer Manipulation sein? (Kostede 1976, S. 154)

Es war also nicht nur die unmittelbare Konfrontation der Studentenbewegung mit der Repressivität des bürgerlichen Staates, der Tanz der Staatsknüppel gewissermaßen (vgl. ebd., S. 153), der die kritischen Geister anregte. Es waren auch und vor allem die ökonomischen und politischen Entwicklungstendenzen zu Beginn der siebziger Jahre, die erheblich dazu beitrugen, daß die diversen Zirkel matxistischer Intellektueller, die nach dem Zerfall der außerparlamentarischen Opposition in der BRD und Westberlin gebildet worden waren, den bürgerlichen Staat theoretisch neu einzuschätzen versuchten.

So erforderte die ökonomische Entwicklung seit der Rezession von 1966 eine theoretische Aufarbeitung. Die - wenn auch nur oberflächliche - Bewältigung dieser Wirtschaftskrise rückte das differenzierte Instrumentarium staatlicher Wirtschaftspolitik zur Krisenregulation und Konjunktursteuerung, allgemein zur Stabilisierung der kapitalistischen Gesell- 
schaftsformation ins Blickfeld der Analyse. Zwar war dieser Staatsinterventionismus keineswegs neueren historischen Datums. Doch die Intensität, mit der seit Ende der sechziger Jahre Probleme ökonomischer und gesellschaftlicher Planung diskutiert wurden, mußte die Fragen nach dem Verhältnis von Politik und Ökonomie, nach den ökonomischen Funktionen des bürgerlichen Staates und nach den Grenzen staatlicher Regulierungstätigkeit auf die Tagesordnung marxistischer Theoriediskussionen setzen (vgl. Classen 1979, S. 2 f.; Altvater/Kallscheuer 1979, S. 8 f.).

Das spezifische am Staatsinterventionismus der siebziger Jahre war die Tatsache, daß er von einer sozialliberalen Koalition mit reformistischem Anspruch betrieben wurde. Wollte man sich von marxistischer Seite mit der Funktionsweise, den Grenzen und Widersprüchen reformistischer Regierungspolitik und den Möglichkeiten einer Gesellschaftsveränderung auf parlamentarischem Wege auseinandersetzen, so schloß dies eine Debatte nahezu aller zentralen strategischen Probleme der Arbeiterbewegung ein. In einem engen Zusammenhang mit diesen Fragen antikapitalistischer Strategie stand die Erörterung der sozialpolitischen Funktionen des bürgerlichen Staates, hatten diese sich doch als ein Instrument der sozialen Integration der lohnabhängigen Bevölkerung in das kapitalistische Gesellschaftssystem erwiesen.

Die hier nur angedeuteten Problemkomplexe, die den marxistischen Staatstheoretikern von den gesellschaftlichen und politischen Verhältnissen in der BRD aufgedrängt wurden und von eminenter strategischer Bedeutung waren, standen auch im Mittelpunkt jenes von Wolfgang Müller und Christel Neusüß verfaßten Aufsatz über die »Sozialstaatsillusion« (vgl. Müller/Neusüß 1971), der den Beginn der spezifisch bundesdeutschen »Ableitungs«Diskussion markierte. In diesem Aufsatz wurden all jene oben genannten Themen angesprochen, die die weitere staatstheoretische Debatte in den siebziger Jahren bestimmen sollten. Und in diesem Aufsatz wurde mit der Betonung der Notwendigkeit einer kategorialen Analyse des bürgerlichen Staates (vgl. ebd., S. 7) auch der Weg vorgezeichnet, von dem sich die bundesdeutschen Marxisten eine Beantwortung aller wesentlichen Fragen erhofften: das methodische Verfahren einer *Ableitung von Form und Funktion des Staates in der kapitalistischen Gesellschaft, das heißt der Versuch einer »begriffliche(n) Entwicklung politischer Grundstrukturen aus der ökonomischen Formation der bürgerlichen Gesellschaft" (Kostede 1976, S. 156).

Der ursprüngliche, von den politischen Ausgangsproblemen diktierte Anspruch der marxistischen Staatsdiskussion zu Beginn der siebziger Jahre, mit Hilfe einer Analyse sozialpolitischer Maßnahmen und staatsinterventionistischer Reformpolitik sowie der Ideologiekritik "revisionistischer« Theorien, einen Beitrag zur Klärung wichtiger strategischer Probleme der Arbeiterbewegung zu leisten, wurde also gleich zu Beginn der Ableitungsdebatte nur höchst unvollständig eingelöst. Im Verlauf der nächsten Jahre entfernten sich die staatstheoretischen Bemühungen marxistischer Intellektueller zunehmend von den erwähnten politisch-strategischen Ausgangspunkten und verloren die historischen Erscheinungsformen des bürgerlichen Staates und aktuelle politische Probleme immer mehr aus den Augen. Die Orientierung an logisch-kategorialen Formanalysen und Versuche, "Staatsableitungen' als begriffslogische Konstrukte zu perfektionieren« (Hirsch 1976, S. 101), gewannen endgültig die Oberhand.

Die Ursache für diese eigentümliche Entwicklung lag in der spezifischen Situation der marxistisch orientierten Linken in der BRD zu Beginn der siebziger Jahre. Parallel zur Staatsdebatte und mit ihr eng zusammenhängend verliefen ja jene Versuche einer »Rekonstruk- 
tion " der Kritik der politischen Ökonomie, durch die viele der während der studentischen Protestbewegung politisierten Intellektuellen sich - nach Jahrzehnten dauernder wissenschaftspolitischer Unterdrückung des Marxismus in der BRD - das Marxsche Werk neu aneignen wollten. Da dieses Unterfangen mit deutscher Gründlichkeit angegangen wurde, mündete die aus dem verständlichen Bedürfnis nach einer exakten »Kapital«-Lektüre entstandene.Marx-Oxtbodoxie schnell in bloßer Quellenexegese und Marx-Philologie. Die hochabstrakte, logisch-kategoriale Orientierung der Ableitungsdiskussion muls zweifellos als eine Erscheinungsform der Befriedigung des Nachholbedarfs an genuiner Marx-Kenntnis gewertet werden. Die negativen Auswirkungen dieser neuen marxistischen Orthodoxie zeigten sich in allzu vielen Ableitungsvarianten als an »Zitierwahn« (Negt 1976, S. 16) grenzende, bloß philologische Zusammenstellungen von »Klassiker«-Aussagen über den bürgerlichen Staat (vgl. Hirsch 1976, S. 99; Altvater/Kallscheuer 1979, S. 8).

Dieser inflationistische Umgang mit »Klassiker«-Zitaten kann mit den Notwendigkeiten, die sich aus dem Fortgang der Theoriebildung ergaben, allein nicht erklärt werden. In einer Situation heilloser Zersplitterung und erbitterter Konkurrenz innerhalb der Linken hatte die begriffslose, weil den Gesamtzusammenhang des Marxschen Werkes und den Stellenwert einzelner Aussagen innerhalb dieses Zusammenhangs völlig ignorierende Aneinanderreihung diverser »Klassiker«-Aussagen, was etwa das Projekt Klassenanalyse (PKA) bis zu grotesker Perfektion betrieb, auch und vor allem die primäre Funktion einer Immunisierung des eigenen Theorieansatzes gegen die Kritik anderer Gruppen. In den innerfraktionellen Auseinandersetzungen der bundesdeutschen Linken jedenfalls wurde die Marxsche Theorie allzu oft für die Legitimierung der eigenen theoretischen Position und qua Theorieproduktion damit zur Stabilisierung der nach dem Zerfall der Studentenbewegung prekären politiscben Identität instrumentalisiert.

Welch verheerende Folgen dies hatte, läßt sich beispielhaft am staatstheoretischen Ansatz des heute unter der Bezeichnung sMarxistische Grupper (MG) firmierenden Intellektuellenzirkels um Herbert L. Fertl und Karl Held ablesen. Die MG betrachtete die Verkehrsformen an der Oberfläche der kapitalistischen Gesellschaft, die Konkurrenz der Individuen, als den Ausgangspunkt der Staatsanalyse. Ausgehend vom "gewöhnlichen« Bewußtsein der Privatsubjekte im Kapitalismus, nämlich ihrem Interesse an der optimalen Nutzung ihrer jeweiligen »Revenuequelle«, schloß die MG auf den $W$ illen der Individuen, sich einer besonderen Instanz zu unterwerfen, die für die Respektierung der Verfolgung ihrer gegensätzlichen Sonderinteressen Sorge zu tragen habe (vgl. MG 1979, S. 8 ff.):

»Neben ihren ökonomischen Geschäften sind sie (die Mitglieder der kapitalistischen Gesellschaft, G.R.) politische Bürger, und wollen die staatliche Herrschaft, weil sie ihren Sonderinteressen nur nachgehen können, indem sie von ihnen auch abstrahieren. Der bürgerliche Staat ist also die Verselbständigung ihres abstrakt freien Willens.« (ebd., S. 8)

Da die MG also die Entstehungsgrundlage des bürgerlichen Staates im - Marx zufolge falschen! - Bewußtsein der Privatsubjekte ortete, von daher die materielle Basis, die in den ökonomischen Gesetzmäßigkeiten der kapitalistischen Gesellschaft wurzelnde Notwendigkeit einer staatlichen Sphäre, für sie kein Thema mehr zu sein schien, mußte sie den Individuen unvermeidlich einen »Willen zur politischen Herrschaft« (ebd., S. 13) unterstellen. Der Schlüssel zum Staatsverständnis der MG, das kaum als Versuch gewertet werden kann, Staatsanalyse materialistisch zu fundieren, sondern eher als moderne Form einer Staatsvertragstheorie angesehen werden muß, liegt zweifellos in der strategischen Orientierung der MG. Denn die Bestimmung des bürgerlichen Staates als Verselbständigung des abstrakt 
freien Willens der in den Mystifikationen der kapitalistischen Konkurrenz befangenen Privatsubjekte lieferte der MG die theoretisch perfekte Legitimation ihrer Existenz als universitärer Intellektuellenzirkel. Wenn nämlich der Staat tatsächlich ein Produkt der Verselbständigung des freien Willens der Individuen ist, dann ist auch seine Abschaffung sowie die Revolutionierung der gesamten kapitalisten Gesellschaft letzlich das Ergebnis des Wollens der Arbeiterklasse. Das MG-Mitglied, sich im Besitz der ganzen Wahrheit wähnend, kann die Entscheidung für und gegen den Kapitalismus nur beeinflussen, indem er den Lohnabhängigen die »richtigen« Argumente zur Verfügung stellt.

In dieser Perspektive erscheint der von der MG betriebene Semirarmarxismus sowie die Herstellung und Verteilung von Flugblättern in denen die Agitationsobjekte die wesentlichen Erkenntnisse der MG verabreicht bekommen, als höchste Form revolutionärer, kommunistischer Praxis. Insofern konsequent, denunziert die MG all jene realen Widerspruchspotentiale, die ihre Grundlage in individueller und kollektiver Betroffenheit durch die Destruktivität der kapitalistischen Gesellschaft haben, sich jedoch nicht direkt auf den Widerspruch von Kapital und Arbeit beziehen, als reformistisch, staatsbejahend und letztlich systemstabilisierend. Dieser zutiefst zynischen Haltung gegenüber »politisierten« und damit angeblich in den demokratischen Staat integrierten Bedürfnis- und Interessenartikulationen, die an einzelnen Mißständen der kapitalistischen Gesellschaft ansetzen (vgl. MG 1980, S. 3 - 16), entspricht die völlige persönliche Folgenlosigkeit der Politik der MG, die in keinerlei Beziehung mit dem privaten Lebenszusammenhang ihrer Mitglieder steht. Was den politischen Realitätsverlust und die geradezu systematische Selbsttäuschung über die tatsächlichen Handlungsmöglichkeiten und Erfolgsaussichten marxistischer Intellektueller angeht, war und ist die MG zweifellos das eindrucksvollste und zugleich abschreckendste Beispiel für die verhängnisvollen Folgen, die die Instrumentalisierung marxistischer Theorieproduktion zur Legitimation bestimmter politisch-strategischer Positionen und damit auch und gerade für die Stabilisierung persönlicher und politischer Identität hatte.

Am Beispiel der MG lässt sich zeigen, welch hohen Stellenwert die Theoriearbeit in der bundesdeutschen Linken während der siebziger Jahre hatte. Oft genug ersetzte sie angesichts politischer Perspektivlosigkeit die vordem durch die Mitgliedschaft in einer Partei oder Organisation oder auch nur durch die Zugehörigkeit zur antiautoritären Bewegung insgesamt konstituierte politische Identität. Die umfassende Kenntnis des Marxschen Werkes kompensierte gewissermaßen die politische Unsicherbeit und Orientierungslosigkeit (vgl. Negt 1976, S. 38), mit jeder theoretischen Kontroverse stand so auch die eigene brüchige politische Identität zur Debatte. Fast unvermeidliche Folge war eine Dogmatisierung der marxistischen Theoriediskussion, die Hypostasierung einzelner Ansätze und Versuche zur Immunisierung dieser Ansätze gegen Kritik.

Im Rahmen der Ableitungs-Debatte führte der sektenhaft-dogmatische Charakter der innerlinken Auseinandersetzung zu einem geradezu »kleinbürgerliche(n) Besitzindividualismus in der Verteidigung von Ableitungsansätzen« (Asseln/Deppe 1977, S. 84). Unter diesen Umständen eines verbissen, zuweilen gar eifersüchtig geführten Streites um Marxens Bart war es nicht weiter verwunderlich, daß die meisten Ableitungsvarianten vor dem Kriterium der Praxisrelevanz marxistischer Staatstheorie kläglich versagten. Das Pathos revolutionärer Stategie verkam zum Ritual, das sich vornehmlich in Vorbemerkungen und Nachworten abspielte und mit den eigenen theoretischen Anstrengungen nur notdürftigt, wenn überhaupt vermittelt war. 
Ein Musterbeispiel für die strategische Beliebigkeit und - daraus resultierend - politische Folgenlosigkeit hochabstrakter Formableitungen bot das PKA. Dieser theoretische Zirkel um Joachim Bischoff brachte es fertig, mit seiner bis heute im wesentlichen unveränderten Staatsableitung (vgl. PKA 1973, S. 69-175; PKA 1976; Bischoff (Hrsg.) 1977, S. 139-170) zwei völlig unterschiedlich strategische Orientierungen zu »begründen«. Doch weder das Bekenntnis des PKA zur DKP als angeblich fortgeschrittensten Teil der Arbeiterbewegung (vgl. PKA 1972, S. 25 ff.) zu Beginn der siebziger Jahre noch das Einschwenken auf eine eurokommunistische Linie (vgl. Bischoff (Hrsg.) 1977, S. 105 ff.; Sozialistische Studiengruppe 1980, S. 188-195) wurde aus den staatstheoretischen Schriften systematisch hergeleitet. Die Strategiebestimmungen des PKA müssen deshalb interpretiert werden als die je verschiedenen Versuche eines Theorie produzierenden Intellektuellenzirkels, seine politische Abstinenz durch das voluntaristische Bekenntnis zu einer politischen Richtung zu überwinden.

Nun darf über der berechtigten Kritik an den strategischen Defiziten der Ableitungs-Debatte nicht vergessen werden, daß auch die hochabstrakte Ableitung bestimmter politischer Grundstrukturen aus der Marxschen Kritik der politischen Ökonomie durchaus ihre Bedeutung in politischen Auseinandersetzungen haben können. Zwei Beispiele sollen diese These belegen.

- Ein wesentlicher Themenkomplex der Ableitungsdebatte war - wie schon erwähnt das Problem des Zusammenhangs von kapitalistischer Akkumulation und zunehmender staatsinterventionistischer Regulierungspolitik und allgemeiner die Frage nach den ökonomischen Funktionen des bürgerlichen Staats überhaupt (vgl. für viele Altvater 1972; Hirsch 1974; Läpple 1973). Die strategische Relevanz dieses Schwerpunktes ist leicht einzusehen, war doch die Analyse der ökonomischen Funktionen des bürgerlichen Staats durch die keynesianische Interventionspolitik der sozialliberalen Koalition auf die Tagesordnung marxistischer Wissenschaftler gesetzt worden. Nur »wenn der Nachweis gelang, daß der Staat in der Wahrnehmung dieser Funktionen umfassenden Beschränkungen unterlag, er folglich auch nicht auf Dauer die zentralen kapitalistischen Krisenmanifestationen und die in ihrem Gefolge beschleunigten Klassenkämpfe latent zu halten vermochte, dann und nur dann waren die strategischen Konzepte der diversen 'politischen Krisentheorien' widerlegbar« (Kostede 1976, S. 170) und konnte an tradierten revolutionären Strategiemustern festgehalten werden.

- Dieses zu Beginn der siebziger Jahre strategisch äußerst bedeutsame Problem der möglichen Grenzen staatlicher Regulierungstätigkeit versuchten auch Bernhard Blanke, Ulrich Jürgens und Hans Kastendiek anzugehen, indem sie auf der Basis ihrer Formableitung ein kategoriales Konzept für die Unterscheidung einer System- und Tätigkeitsgrenze der Staatsaktivitäten entwickelten (vgl. Blanke u.a. 1974, S. 89-100). Welch große politische Bedeutung ein derartiger Analyseansatz im Kontext der Auseinandersetzungen um die Einschätzung der von der bundesdeutschen Sozialdemokratie damals propagierten Reformpolitik hatte, zeigten nicht zuletzt die breite Rezeption des Aufsatzes von Blanke und seinen Kollegen bei den Jungsozialisten und die dadurch ausgelösten innerverbandlichen Kontroversen (vgl. Heimann 1975, S. 215-222).

Doch - wie schon angedeutet - derartige Hinweise auf die strategischen Implikationen marxistischer Staatstheorie waren in der vornehmlich logisch-kategorialen Argumentation der Ableitungstheoretiker allzu selten. Allein schon die Tatsache, daß Blanke, Jürgens und Kastendiek den eigentlich banalen und für Marxisten selbstverständlichen Umstand glaub- 
ten betonen zu müssen, daß materialistische Staatsanalysen sich immer auf die aktuellen Probleme der Arbeiterbewegung zu beziehen hätten, wollten sie zur praktischen Einschätzung der Maßnahmen des bürgerlichen Staates und zur sozialistischen Transformation der kapitalistischen Gesellschaft beitragen (vgl. Blanke u.a. 1974, S. 58), wirft ein bezeichnendes Licht auf das offenkundige strategische Defizit der Ableitungsdebatte.

Die Abstraktheit und Realitätsferne der Ableitungsdiskussion, die Konzentration auf die Perfektionierung begriffslogischer Konstrukte, der zunehmende Verlust eines Bezugs auf praktisch-politische Fragen werden nur dann verständlich, wenn man bedenkt, daß die politischen Machtverhältnisse in der BRD etwas anderes als eine antiinstitutionelle Opposition des Linken nie zuließ (vgl. Altvater/Kallscheuer 1979, S. 9). Isoliert von einer weitgehend sozialdemokratisch und gewerkschaftlich integrierten Arbeiterklasse, aber auch von anderen sozialen Oppositionsansätzen wie der Frauen- und Ökologiebewegung, beschränkten sich die marxistischen Intellektuellen auf eine im ideologiekritiscben Sinne destruktive Analyserichtung (vgl. ebd.). Das heißt, »Staatstheorie wurde (...) primär als Analyse der Grenzen des Staates begriffen« (ebd., S. 8), weil die bundesdeutsche Linke »nicht in der politischen Verantwortung stand und daher auch keine positiven Konzeptionen von Politik vorzulegen brauchte.« (Ebd., S. 9)

Wie verhängnisvoll, weil politisch desorientierend, die bloß ideologiekritisch und abstraktkategorial argumentierende Ableitungsdebatte für die Linke in der BRD war, erwies sich spätestens angesichts zunehmender staatlicher Repression Mitte der siebziger Jahre. Als die politische Entwicklung die Frage nach dem praktischen Verhalten der Linken gegenüber dem bürgerlichen Staat auf die Tagesordnung setzte, zeigte sich, wie wenig die Staatsdiskussion in den siebziger Jahren zu einer Antwort auf die Kernfragen antikapitalistischer Strategie und zur Bewältigung der Existenzprobleme der Sozialisten in der BRD beigetragen hatte (vgl. Wolf 1979, S. 33), weil sie zu handlungsorientierenden Anleitung emanzipatorischer Prozesse gänzlich untauglich war.

Die seit Mitte der siebziger Jahre andauernde Krise der kapitalistischen Wirtschaft im Weltmaßstab förderte jedoch die Einsicht in die politische Folgenlosigkeit und strategische Perspektivlosigkeit der Ableitungsdebatte. In dieser Krise wurde nämlich offenkundig, $\gg$ daß sich die revolutionsstrategischen Erwartungen hinsichtlich der Konstituierung und Radikalisierung eines proletarischen Klassenbewußtseins, die fast alle Marxisten an die kapitalistische Krise knüpften, keineswegs erfüllten.«(Redaktion 'Prokla' 1979, S. 6). Nachdem die gesellschaftliche Realität derart nachdrücklich auf die Frage nach der Angemessenheit überkommener maxistischer Theorie-und Strategiemuster aufmerksam gemacht hatte, setzten Denk- und Lernprozesse bei all jenen marxistischen Theoretikern ein, die noch nicht jegliche Sensibilität für spezifische gesellschaftsstrukturelle Problemkonstellationen und die darin implizierten Probleme sozialistischer Politik verloren hatten. Die Forschungsstrategie einer Ableitung des bürgerlichen Staates stand in der Folgezeit - etwa ab 1977 - nicht mehr im Zentrum der analytischen Bemühungen marxistischer Staatstheoretiker, die Staatsdiskussion in der BRD entwickelte sich vielmehr recht uneinheitlich. Folgende Tendenzen zeichnen sich dabei ab:

- Einige wenige marxistische Wissenschaftler versuchen, die bislang fehlenden Vermittlungsglieder zwischen der hochabstrakten Formanalyse des bürgerlichen Staates und der empirischen Untersuchung konkreter politischer Prozesse zu erarbeiten, indem sie systematisch an die Ergebnisse der Ableitungsdebatte anknüpfen. Sowohl Dieter Sauers Operationalisierung verschiedener Ableitungsansätze für eine empirisch orientierte Analyse des 
Staatsapparates (vgl. Sauer 1978) als auch Bernhard Blankes Bemühungen um die kontinuierliche Weiterentwicklung eines staatstheoretischen Ansatzes (vgl. Blanke 1976, 1977, 1979, 1980) zeigen deutlich, daß die Formanalyse des bürgerlichen Staates bei einer realistischen Einschätzung ihrer erkenntnistheoretischen Grenzen durchaus die Basis für eine fruchtbare Forschungsstrategie abgeben kann.

- In einem gewissen Gegensatz zu den verschiedenen Versuchen, die Ableitungsdebatte in je unterschiedliche Richtungen weiterzuentwickeln, stehen jene Arbeiten marxistischer Wissenschaftler, die die Frage nach einer möglichen »Neustrukturierung « bürgerlicher Herrschaft im Gefolge der ökonomischen Krise seit etwa Mitte der siebziger Jahre stellen. Charakteristisch für diese Entwicklungslinie ist die Suche nach einer Erklärung für die Tatsache, $»$ daß sich die Herrschaftsorganisation in der Bundesrepublik auch in der gegenwärtigen Krise und trotz aller Legitimations- und Steuerungsschwächen als sehr stabil erwiesen hat (Kastendiek 1980, S. 82). Zu nennen sind in diesem Zusammenhang etwa Elmar Altvaters Untersuchung der politischen Implikationen der »Austerity-Politik« in Westeuropa (vgl. Altvater 1978), Hans Kastendieks Bemühung, einige Aspekte der KorporativismusDiskussion für die Analyse staatlicher Strukturen und politischer Prozesse in der BRD fruchtbar zu machen (vgl. Kastendiek 1980), und Joachim Hirschs Analyse der Wandlung der BRD hin zu einem Sicherheitsstaat (vgl. Hirsch 1980). Vor allem Hirsch bemüht sich ganz offensichtlich, die strategische Dimension materialistischer Staatstheorie zu reaktivieren und die politische Folgenlosigkeit der Ableitungsdebatte zu überwinden, indem er sich eingehend mit der Problematik der »neuen sozialen Bewegungen« beschäftigt und die Frage nach der Bedeutung der Frauen-, Alternativ- und Ökologiebewegung für die revolutionäre Veränderung hochentwickelter kapitalistischer Gesellschaften stellt (vgl. ebd., S. 132168).

- Die Bemühung, die politischen Defizite der Ableitungsdebatte zu beheben und die strategische Relevanz marxistischer Staatstheorie zu vergrößern, kennzeichnet auch jene Versuche, analytische Konzepte italienischer und französischer Provenienz für die bundesdeutsche Staatsdebatte fruchtbar zu machen (vgl. etwa Arbeitskreis Westeuropäische Arbeiterbewegung (Hrsg.) 1979; Jäger 1980 a und 1980 b). Von Begriffen wie dem des »historischen Blocks an der Macht« oder der »bürgerlichen Hegemonie« über die Arbeiterklasse, wie sie vor allem von Antonio Gramsci geprägt wurden, erhoffen sich am "Eurokommunismus " orientierte Autoren theoretische Fortschritte bei der anstehenden Analyse der konkreten politischen Kräfteverbältnisse in der BRD und Anregungen für die Entwicklung erfolgversprechender sozialistischer Strategiekonzeptionen.

\section{Was kann man aus der bisherigen Entwicklung der marxistiscben Statstbeorie in der $B R D$ lernen?}

Läßt man die - hier nur knapp skizzierte - bisherige Entwicklung der marxistischen Staatstheorie in der BRD noch einmal Revue passieren, so muß man zweifellos feststellen, daß von einem kontinuierlichen Theoriebildungsprozess, der ein Aneinanderknüpfen der verschiedenen Ansätze und eine Ergänzung der wechselseitigen Leerstellen ermöglicht hätte, nicht die Rede sein kann. Was diese Entwicklung vielmehr kennzeichnet, sind ganz offensichtlich ihre Brüche.

Das in der Ära Adenauer vorherrschende und von Wolfgang Abendroth repräsentierte Pa- 
radigma linker Gesellschaftskritik, die Konfrontation von Verfassungsnorm und Verfassungswirklichkeit erschien der sich in der antiautoritären Studentenbewegung politisierenden Intelligenz zu legalistisch und angesichts der angeblich faschistoiden Tendenzen in der BRD zu illusorisch, als daß es eine radikale gesellschaftsverändernde Praxis hätte anleiten können. Dabei hätten gerade die kritischen Studenten für eben diese Praxis von Abendroth lernen können, wie wichtig es ist, die bürgerliche Demokratie als Aktionsterrain von Oppositionsbewegungen zu nutzen und wie entscheidend für den Erfolg derartiger Bewegungen die realistische Einschätzung der jeweiligen Kräftekonstellationen ist? .

Die Studentenbewegung hielt dies - zumindest was ihren konsequent antiautoritären Flügel betrifft - für unnötig und stützte sich in ihrer Theoriebildung auf verschiedene, zweifellos »radikalere Theorieversatzstücke aus dem Umkreis der Kritischen Theorie. Wie wir bereits gesehen haben, lieferte sie damit den späten, aber endgültigen Beweis für die strategische Perspektivlosigkeit der Frankfurter Schule. Das realpolitische Scheitern der studentischen Protestbewegung bedeutete deshalb mit Recht einen theoretischen Neubeginn und den notwendigen Versuch, dem Verhältnis von bürgerlichem Staat und kapitalistischer Gesellschaft analytisch auf den Grund zu gehen.

Leider ging mit dieser Distanzierung von als falsch erkannten Theoriekonzepten eine pauschale Kritik der Studentenbewegung als »kleinbürgerlich« einher, die die positiven Aspekte des antiautoritären Protestes vollkommen negierte. Die nachdrückliche Insistenz auf det praktischen Relevanz von Theoriebildungsprozessen, das primäre Interesse an einer radikalen gesellschaftsverändernden Praxis, die Betonung individueller Selbstveränderung_und Emanzipation in einem kollektiven politischen Lernprozeß - all diese wesentlichen Momente der Studentenbewegung gingen mit der Hinwendung zu K-Gruppen-Sektierertum und dogmatischem Seminarmarxismus in Vergessenheit oder wurden - ihrer politischen Stoßrichtung weitgehend beraubt - in ein spontaneistisch-subkulturelles Ghetto abgedrängt. Die folgende, die siebziger Jahre weitgehend prägende Marx-Orthodoxie brachte Theoriediskussionen von in der BRD bis dahin nicht gekanntem theoretischen Niveau und - was die Staatstheorie betrifft - durchaus bemerkenswerte Ergebnisse im Hinblick auf die Analyse politischer Grundstrukturen der bürgerlichen Gesellschaft. Bezahlt wurde dieser Rückzug in den akademischen Elfenbeiturm marxistischer Wissenschaft bekanntermaßen damit, daß der Marxismus in der BRD seine strategische Relevanz einbüßte, politisch folgenlos blieb, angesichts zunehmender staatlicher Repression keinerlei Handlungsanleitung bot, ja sogar politisch desorientierend wirkte.

Was also können wir aus der bisherigen Entwicklung der marxistischen Staatstheorie in der BRD lernen? Zunächst und vor allem muß die bundesdeutsche Linke ihre eklatante Geschichtslosigkeit im Hinblick auf ihre eigene theoretische, organisatorische und politische Tradition ablegen. Sie muß lernen, die theoretischen Defizite und die politisch-strategischen Fehleinschätzungen der Vergangenheit produktiv aufzuarbeiten, anstatt sich bloß umstandslos von ihnen zu distanzieren. Für den Bereich der Staatstheorie bedeutet dies konkret: Die Linke in der BRD könnte heute - nach rund 30 Jahren mehr oder minder intensiver Theoriediskussionen - an einem Punkt angelangt sein, an dem marxistische Staatstheorie den klaren Blick eines Wolfgang Abendroth für die realistische Einschätzung politischer Kräfteverhältnisse mit der politischen Radikalität der Studentenbewegung und dem theoretischen Niveau der Ableitungsdebatte verbindet. Daß dem keineswegs so ist, wir uns vielmehr mühsam staatstheoretische Orientierungspunkte für die achtziger Jahre erarbeiten müssen, wirft ein bezeichnendes Licht auf den Grad unserer Lern(un)fähigkeit. 
Was heißt das im Hinblick auf die Wiedergewinnung der strategischen Relevanz marxistischer Staatstheorie? Wenn es richtig ist, daß das entscheidende Bewährungskriterium revolutionärer Theorie die Praxis der Gesellschaftsveränderung ist, dann muß die »Beantwortung der Frage, wie im bürgerlichen Staat zu agieren und dieser in emanzipatorischer Perspektive aufzuheben« (Redaktion 'Prokla' 1979, S. 8) ist, in das Zentrum materialistischer Staatsanalyse rücken. Wollen die Marxisten in der BRD dieses Problem ernsthaft und vorurteilslos angehen, so wetden sie nicht umhin kommen, Abschied von einigen tradierten Theorie- und Strategiemustern zu nehmen. ${ }^{10}$ Sie werden vor allem der Frage nachzugehen haben, warum einerseits die Hoffnung auf die historische Mission des Proletariats trotz verschärfter Wirtschaftskrise bisher unerfüllt blieb, wie andererseits die vielfältigen Formen neuer sozialer Bewegungen mit emanzipatorischen Ansprüchen politisch-strategisch einzuschätzen sind. Die Beantwortung beider Fragen setzt ein Problem auf die Tagesordnung, dem marxistische Theoretiker bislang fast systematisch aus dem Wege gegangen sind: die theoretisch stringente und empirisch fundierte Bestimmung des Vermittlungszusammenbanges zwischen der objektiven Struktur der kapitalistischen Gesellschaft und dem subjektiven Bewußtsein der Individuen. Wollen die marxistischen Staatstheoretiker in der BRD nicht weiterhin der Stabilität bürgerlicher Herrschaft in ökonomischen Krisenzeiten analytisch hilflos gegenüberstehen, dann muß die Ausgrenzung aller Fragen der »Subjektivität« beendet werden, die die Geschichte des Marxismus bislang kennzeichnet (vgl. BlankełSchäfer) 1979, S. 44), müssen vor allem jene offenbar äußerst tragfähigen sozialen und psychischenMechanismen, die die Reproduktion der kapitalistischen Gesellschaft auch in Krisenzeiten gewährleisten, sorgfältig untersucht werden (vgl. Blanke/Schäfer 1979, S. 43 f.; Hirsch 1980, S. 137 f.). Erst wenn die marxistische Theorie dieses Problem konkret angeht, kann sie zu einer "Theorie des Übergangs" (vgl. Blanke/Schäfer 1979, S. 45) mit strategischem Stellenwert werden, weil sie dann in der Lage sein wird, auch in einer als Jahrzehnte währenden Lernprozeß zu verstehenden »Revolution«(vgl. ebd., S. 44) längerfristig handlungsorientierend zu wirken.

\section{Anmerkungen}

1 Für die ausführliche Darstellung einzelner Theorievarianten und die Analyse des politisch-gesellschaftlichen Hintergrunds ihrer Entwicklung verweise ich auf mein Buch »Die Entwicklung der marxistischen Staatstheorie in der Bundesrepublik« (Campus-Verlag, Frankfurt/New York 1981). Aus diesem Grunde verzichte ich im folgenden auf ausführliche Zitate und Literaturhinweise.

2 Einen Überblick über die Kritik an Abendroths Position gibt Perels (1974, S. $150 \mathrm{ff}$.).

$3 \mathrm{Zu}$ nennen wären neben Abendroth auf der einen und Horkheimer und Adorno auf der anderen Seite allenfalls noch Leo Kofler und Jürgen Habermas. Während der marxistische Einzelgänger Kofler weitgehend unbeachtet und einflußlos blieb, war Habermas' sozialphilosophische Untersuchung des »Strukturwandels der Öffentlichkeit« (1962) von nicht zu unterschätzender Bedeutung für die weitere Theoriebildung der bundesdeutschen Linken. In unserem thematischen Zusammenhang bringt eine ausführliche Analyse jedoch nichts Neues: Gleichermaßen beeinflußt von A bendroth und der Frankfurter Schule bemühte sich Habermas zum einen um einen prinzipiell kritischen theoretischen Zugriff auf die bürgerliche Gesellschaft, behielt dabei aber immer den 
Stellenwert der politischen Freiheitsrechte der parlamentarischen Demokratie im Auge. Während in diesem Aspekt von. Habermas' Theoriebildung vor allem der Einfluß Abendroths spürbar wurde, so teilte Habermas auf der anderen Seite das strategische Defizit der Kritischen Theorie. Der emphatischen Akzentuierung seines Anspruches, einen Weg zu vernünftig freier Selbstbestimmung weisen zu wollen, stand die Unmöglichkeit einer politisch-strategischen Operationalisierung seines Emanzipationskonzepts gegenüber (vgl. Tuschling 1978, S. 436, 448 f., 456; Kallscheuer 1981, S. $172 \mathrm{ff}$.).

4 Zur genauen Analyse des gesellschaftsstrukturellen Bedingungszusammenhangs der studentischen Protestbewegung vgl. Rudi 1978, S. 4 - 41.

5 Wichtig für das Staatsverständnis der antiautoritären Bewegung war einerseits der 1940 entstandene Aufsatz »Der autoritäre Staat《 von Max Horkheimer (vgl. Horkheimer 1967, S. 41 - 80), andererseits Herbert Marcuses erstmals 1964 in den USA erschienene Studie über den »eindimensionalen Menschen« (vgl. Marcuse 1967).

6 Zur Strategiedebatte in der Neuen Linken vgl. ausführlich Rudel 1978, S. 55 - 58 und S. 66 - 82.

7 Die im folgenden zu analysierende Problematik des Staatsverständnisses der antiautoritären Studentenbewegung wirft auch ein bezeichnendes Licht auf die politisch-strategischen Defizite der Kritischen Theorie, auf deren Theoremen studentische Theoretiker wie Dutschke und Krahl im wesentlichen aufbauten. Nachdem die kritische Intelligenz die radikale Kulturkritik der Frankfurter Schule und den Totalitarismusverdacht eines Herbert Marcuse systematisch ernstzunehmen begann und versuchte, praktische Konsequenzen aus der Kritischen Theorie zu ziehen, zeitigte deren staatstheoretisches und politisch-strategisches Defizit für die studentische Protestbewegung fatale Folgen.

8 Insofern müssen auch die terroristischen Aktionen der RAF und der Bewegung 2. Juni als - ungewollte - strategische Spätfolgen des Staatsverständnisses der antiautoritären Bewegung und des darin implizierten Faschismusverdachts angesehen werden.

9 Es mutet wie ein Treppenwitz der Geschichte an und ist bezeichnend für die Verdrängungsleistungen der bundesdeutschen Linken, wenn sie heute diese Probleme, die immer im Mittelpunkt der Theorieproduktion Wolfgang Abendroths standen (vgl. Seifert 1977, S. 244 f.), mit analytischen Konzepten italienischer Provenienz zu lösen versucht. Damit soll durchaus kein negatives Urteil über die Legitimität des derzeitigen Gramsci-Booms und die forschungsstrategische Brauchbarkeit seiner Kategorien gefällt werden. Die Aktualität der Gramsci-Diskussion - und auch die Renaissance austromarxistischer Denker wie Otto Bauer (vgl. Albers u. a. (Hrsg.) 1979) - sind jedoch ein deutlicher Hinweis auf die Unfähigkeit der Marxisten in der BRD, die positiven Aspekte einer bestimmten Theorievariante wie etwa der von Abendroth - ungeachtet aller berechtigten Kritik - bei der Weiterentwicklung der Theoriebildung in einem dialektischen Sinne »aufzuheben«.

10 Einen knappen und sehr informativen Überblick über die verschiedenen Dimensionen der $»$ Krise des Marxismus gibt Redaktion 'Prokla' 1979, S. 6 - 10.

\section{Literatur}

Abendroth; Wolfgang: Das Grundgesetz. Eine Einführung in seine Probleme, Pfullingen 1966 ders.: Arbeiterklasse, Staat und Verfassung. Materialien zur Verfassungsgeschichte und Verfassungstheorie der Bundesrepublik, Frankfurt/Köln 1975

ders.: Ein Leben in der Arbeiterbewegung. Gespräche, aufgezeichnet und herausgegeben von B. Dietrich und J. Perels, Frankfurt 1976

Agnoli, Johannes: Die Transformation der Demokratie; in: ders. / Peter Brückner: Die Transformation der Demokratie, Frankfurt 1968

ders.: Thesen zur Transformation der Demokratie und zur außerparlamentarischen Opposition; in: Neue Kritik 47 (1968), S. 24 - 33 
Albers, Detlev/Josef Hindels/L. Lombardo Radice (Hrsg.): Otto Bauer und der Dritte Weg. Die Wiederentdeckung des Austromarxismus durch Linkssozialisten und Eurokommunisten, Frankfurt/New York 1979

Altvater, Elmar: Zu einigen Problemen des Staatsinterventionismus; in: Prokla 3 (1972), S. 1 - 53

ders.: Politische Implikationen der Krisenbereinigung. Überlegungen zu den Austerity-Tendenzen in Westeuropa; in Prokla 32 (1978), S. $43-72$

ders. / Otto Kallscheuer: Reaktionen auf eine Provokation. Wie die Linke in Italien die Krise des Marxismus diskutiert; in: dies. (Hrsg.): Den Staat diskutieren. Kontroversen über eine These von Althusser, Berlin 1979, S. 7 - 40

Arbeitskreis Westeuropäische Arbeiterbewegung (Hrsg.): Eurokommunismus und marxistische Theorie der Politik. Argument-Sonderband 44 (1979)

Asseln, Heiko/Frank Deppe: Die »Staatsfrage und die Strategie der Arbeiterbewegung; in: Probleme der materialistischen Staatstheorie. Staat und Monopole (II), Argument-Sonderband 16 (1977), S. $84-132$

Bernd, Heide: Die Suche nach dem »revolutionären Subjekt«; in: Neue Kritik 45 (1968), S. 94 - 99 Bischoff, Joachim (Hrsg.): Marxismus und Staat. Einführung in die marxistische Staatstheorie, Hamburg/Berlin 1977

Blanke, Bernhard: Entscheidungsanarchie und Staatsfunktionen. Zur Analyse der Legitimationsprozesse im politischen System des.Spätkapitalismus; in: Rolf Ebbighausen: Bürgerlicher Staat und politische Legitimation, Frankfurt 1976, S. $188-216$

ders.: Formen und Formwandel des politischen Systems in der bürgerlichen Gesellschaft; in: Volkhard Brandes u. a. (Hrsg.): Handbuch, 5. Staat, Frankfurt/Köln 1977, S. $121-160$

ders.: Demokratische Verfassung und »Verfassungsfeinde«. Die Spaltung der Demokratie. Zur Regelung des Zugangs zum öffentlichen Dienst in der Bundesrepublik seit 1972, Hannover 1979

ders.: Reproduktion des Kapitals als Verfassungsproblem. Die Unternehmermitbestimmung in der grundrechtlichen Sicht des Bundesverfassungsgerichts: politischer Konsens und ökonomische Stabilität als »Funktionsbedingung« ihrer Verfassungsmäßigkeit; in: Prokla 38 (1980), S. $63-80$

ders. / Ulrich Jürgens / Hans Kastendiek: Zur, neueren marxistischen Diskussion über die Analyse von Form und Funktion des bürgerlichen Staates. Überlegungen zum Verhältnis von Politik und Ökonomic; in: Prokla 14/15 (1974), S. 51 - 102

ders. / Gert Schäfer: Krise der Linken - Krise des Marxismus; in: Prokla 36 (1979). S. 35 - 48

Classen, Wolfgang-Dieter: Probleme einer materialistischen Analyse des bürgerlichen Staates, Frankfurt 1979

Dubiel, Helmut: Wissenschaftsorganisation und politische Erfahrung. Studien zur frühen Kritischen Theorie, Frankfurt 1978

Dutschke, Rudi: Die Widersprüche des Spätkapitalismus, die antiautoritären Studenten und ihr Verhältnis zur Dritten Welt; in: Bergmann u. a.: Rebellion der Studenten oder Die neue Opposition; Reinbek bei Hamburg 1968, S. $33-93$

Habermas, Jürgen: Strukturwandel der Öffentlichkeit. Untersuchungen zu einer Kategorie der bürgerlichen Gesellschaft, Neuwied/Berlin 1962

Heimann, Horst: Theoriediskussion in der SPD. Ergebnisse und Perspektiven, Frankfurt/Köln 1975

Hirsch, Joachim: Staatsapparat und Reproduktion des Kapitals, Frankfurt 1974

ders.: Bemerkungen zum theoretischen Ansatz einer Analyse des bürgerlichen Staates; in: Gesellschaft. Beiträge zur Marxschen Theorie 8/9 (1976), S. 99 - 149

ders.: Der Sicherheitsstaat. Das 'Modell Deutschland'. Seine Krise und die neuen sozialen Bewegungen, Frankfurt 1980

Horkheimer, Max: Autoritärer Staat. Die Juden und Europa. Vernunft und Selbsterhaltung. Aufsätze 1939 - 1941, Amsterdam 1967

Jäger, Michael: Ökonomie und Politik des sozialliberalen Korporatismus; in: Sozialliberalismus oder rechter Populismus? Hegemonie und Politik in der Bundesrepublik Deutschland. Argument-Sonderband $51(1980$ a), S. $110-143$ 
ders.: Marxistische Staatstheorie; in: Das Argument 124 (1980) b, S. 795 - 808

Jay, Martin: Dialektische Phantasie. Die Geschichte der Frankfurter Schule und des Instituts für Sozialforschung 1923 - 1950, Frankfurt 1976

Kallscheuer, Otto: Auf der Suche nach einer politischen Theorie bei Jürgen Habermas; in: Ästhetik und Kommunikation 45/46 (1981), S. 171 - 182

Kastendiek, Hans: Neokorporativismus? Thesen und Analyse-Konzepte in der westdeutschen Diskussion und in der internationalen $\nu$ corporatism«-Debatte; in: Prokla 38 (1980), S. 81 - 106

Kostede, Norbert: Die neuere marxistische Diskussion über den bürgerlichen Staat. Einführung - Kritik - Resultate; in: Gesellschaft. Beiträge zur Marxschen Theorie 8/9 (1976), S. 150 - 196

Krahl, Hans-Jürgen: Konstitution und Klassenkampf. Zur historischen Dialektik von bürgerlicher Emanzipation und proletarischer Revolution. Schriften, Reden und Entwürfe aus den Jahren 1966 1970, Frankfurt 1971

Kraushaar, Wolfgang: Thesen zum Verhältnis von Alternativ- und Fluchtbewegung. Am Beispiel der frankfurter scene; in: ders. (Hrsg.): Autonomie oder Ghetto? Kontroversen über die Alternativbewegung, Frankfurt 1978, S. 8 - 67

Krippendorf, Ekkehard: Politikwissenschaft und Außerparlamentarische Opposition; in: Gerhard Lehmbruch u. a. (Hrsg.): Demokratisches System und politische Praxis, München 1971, S. 89 - 123

Krüger, Helga: Zur gesellschaftlichen Funktion der Soziologie; in: Jürgen Klüver/Friedrich O. Wolf (Hrsg.): Wissenschaftskritik und sozialistische Praxis. Konsequenzen aus der Studentenbewegung, Frankfurt 1973, S. $116-136$

Kukuck, Margareth: Student und Klassenkampf. Studentenbewegung in der BRD seit 1967, Hamburg 1974

Läpple, Dieter: Staat und allgemeine Produktionsbedingungen. Grundlagen zur Kritik der Infrastrukturtheorien, Berlin 1973

Lefevre, Wolfgang: Ursachen und Konsequenzen des 2. Juni; in: Neue Kritik 42/43 (1967), S. 4 - 14

Marcuse, Herbert: Der eindimensionale Mensch. Studien zur Ideologie der fortgeschrittenen Industriegesellschaft, Darmstadt/Neuwied 1964

Marxistische Gruppe: Der bürgerliche Staat; in: Resultate der Arbeitskonferenz 3 (1979)

dies.: Die Bundesrepublik Deutschland 1980 - und was Marxisten in den 80er Jahren an ihr zu ändern haben; in: Resultate 1 (1980)/Neufassung

Mirbach, Thomas: Kritik der Herrschaft. Zum Verhältnis von Geschichtsphilosophie, Ideologiekritk und Methodenreflexion in der Gesellschaftstheorie Adornos, Frankfurt/New York 1979

Müller, Wolfgang/Christel Neusüß: Die Sozialstaatsillusion und der Widerspruch von Lohnarbeit und Kapital; in: Prokla Sonderheft 1 (1971), S. 7 - 70

Negt, Oskar: Politik als Protest. Reden und Aufsätze zur antiautoritären Bewegung; Frankfurt 1971

ders.: Überlegungen zu einer kritischen Lektüre der Schriften von Marx und Engels; in: Theorie und Organisation 1 (1976), S. 5 - 40

Nirumand, Baham: Die Avantgarde der Studenten im internationalen Klassenkampf; in: Kursbuch 13 (1968), S. 1 - 17

Perels, Joachim: Legalität und sozialistische Strategie. Zur verfassungstheoretischen Position Wolfgang Abendroths; in: Kritische Justiz 2 (1974), S. 147 - 156

Projekt Klassenanalyse: Leninismus - neue Stufe des wissenschaftlichen Sozialismus? Zum Verhältnis von Marxscher Theorie, Klassenanalyse und revolutionärer Taktik bei W. I. Lenin, Berlin 1972

dass.: Materialien zur Klassenstruktur der BRD. Erster Teil: Theoretische Grundlagen und Kritiken, Berlin 1973

dass.: Thesen zum Verhältnis von bürgerlicher Gesellschaft und Staat; in: Beiträge zum wissenschaftlichen Sozialismus 1 (1976), S. 83 - 101

Reiche, Reimut: Hat der autoritäre Staat der BRD eine Massenbasis? in: Detlev Claussen / Regine Dermitzel (Hrsg.): Universität und Widerstand. Versuch einer politischen Universität in Frankfurt, Frankfurt 1968, S. $21-31$

Redaktion 'Prokla': Was heißt Krise des Marxismus? in: Prokla 36 (1979), S. 1 - 10 
Rudel, Gerd: Studentenbewegung und Rätedemokratie. Demokratietheoretische Aspekte der Renaissance der Räteidee in der Neuen Linken, München 1978 (unveröff. Mag. Arb.)

ders.: Die Entwicklung der marxistischen Staatstheorie in der Bundesrepublik, Frankfurt/New York 1981

Sauer, Dieter: Staat und Staatsapparat. Ein theoretischer Ansatz, Frankfurt/New York 1978

Seifert, Jürgen: Wolfgang Abendroth und die SPD; in: Wolf-Dieter Narr (Hrsg.): Auf dem Weg zum Einparteienstaat, Opladen 1977, S. 242 - 249 